\title{
A powerful diagnostic tool of analytic solutions of ordinary second-order linear homogeneous differential equations
}

\section{Dimitris M Christodoulou ${ }^{1 *}$ and Qutaibeh D Katatbeh²}

\section{"Correspondence:}

dimitris_christodoulou@uml.edu

${ }^{1}$ Department of Mathematical

Sciences, University of

Massachusetts Lowell, Lowell, MA 01854, USA

Full list of author information is available at the end of the article

\begin{abstract}
Very few of the ordinary second-order linear homogeneous (OSLH) differential equations are known to possess analytic, closed-form solutions and there is no general theory to predict whether such solutions exist in each particular case. In this work, we present a powerful new method for the discovery of closed-form solutions in the entire class of the OSLH differential equations. A sufficient condition for the existence of such solutions is that the equations can be transformed to forms with constant coefficients and we show that two predictors exist, a nonlinear first-order Bernoulli equation with index $n=3 / 2$ and a nonlinear second-order equation. There is no need to solve these equations, only to verify them based on the contents of a given OSLH equation. Because the predictors can be verified quite easily in all cases, we believe that this methodology is an important new diagnostic tool for studying all the equations of mathematical physics that belong to the OSLH class.
\end{abstract}

MSC: $34 \mathrm{~A} 25 ; 34 \mathrm{~A} 30$

Keywords: second-order linear differential equations; analytical theory; transformations

\section{Introduction}

The ordinary second-order linear homogeneous (OSLH) differential equations encountered in physics and applied mathematics problems have the general form [1-4]

$$
y^{\prime \prime}+b(x) y^{\prime}+c(x) y=0
$$

where primes denote derivatives with respect to the independent variable $x$ and $b(x)$ and $c(x)$ are generally functions of $x$. Although some analytic solutions are known in closed form for a few specific equations, no general analytic method of solution currently exists. In [4], we found closed-form solutions for the Cauchy-Euler, Riemann-Weber, Chebyshev, and CDOS equations. In these equations, we have identified a common trend that allows us to investigate all OSLH equations analytically: in all four cases, a transformation of the independent variable $x \rightarrow t$ is used in equation (1) that results in a coefficient of the $y(t)$ term that equals unity, viz.

$$
t=\int \sqrt{c(x)} d x \quad \Rightarrow \quad \ddot{y}+\widehat{b}(x) \dot{y}+y=0,
$$

(c) The Author(s) 2017. This article is distributed under the terms of the Creative Commons Attribution 4.0 International License (http://creativecommons.org/licenses/by/4.0/), which permits unrestricted use, distribution, and reproduction in any medium, provided you give appropriate credit to the original author(s) and the source, provide a link to the Creative Commons license, and indicate if changes were made. 
where dots represent derivatives with respect to $t$. Then the new differential equation either shows a constant coefficient $\widehat{b}$ as well, or another transformation to its canonical form produces a constant coefficient. In either one of these cases, the resulting equation can easily be solved analytically. This method stands in contrast to the general transformation to the canonical form: it transfers the dependence on the variable $x$ to the coefficient of $\dot{y}$, precisely the term that the canonical transformation strives to eliminate.

In this work, we apply the same methodology to the general form (1) and we obtain a powerful new diagnostic tool (a 'predictor') that detects whether analytic solutions exist for any choice of the coefficients $b(x)$ and $c(x)$. In the case of constant $\widehat{b} \equiv B$ in equation (2), the predictor is a first-order nonlinear Bernoulli equation $[5,6]$ with index $n=3 / 2$, viz.

$$
c^{\prime}+2 b c=2 B c^{3 / 2},
$$

which $b(x)$ and $c(x)$ must satisfy. In the case of the transformation of equation (2) to its canonical form, the predictor is a complicated second-order equation that we write as a system of two first-order equations.

We derive the predictor equations in Section 2, and we apply the method to some wellknown equations of applied mathematics in Section 3. Finally, we summarize and discuss our results in Section 4.

\section{Discovery of closed-form solutions}

Consider the general form (1) of all OSLH differential equations. The change of independent variable $t=\int \sqrt{c(x)} d x$ recasts this equation to the form given in equation (2), where in particular

$$
\widehat{b}(x) \equiv \frac{1}{\sqrt{ } c}\left(b+\frac{c^{\prime}}{2 c}\right)
$$

where $c^{\prime}=d c / d x$. In the specific cases that we investigate below, we are interested in the existence of real solutions of the differential equations in the $L^{2}(a, b)$ Banach space, although the analysis can be extended to the complex domain as well. Here $(a, b)$ is an interval on the real axis that includes the initial conditions and avoids any singularities. Furthermore, the coefficients $b(x)$ and $c(x)$ are assumed to be continuous in $(a, b)$ and at least twice differentiable everywhere in the interval. In what follows, we use capital letters to indicate various constants.

\subsection{Case $1: \widehat{b}=$ const. $\equiv B$ in equation (2)}

For $\widehat{b}$ equal to a constant $B$, equation (2) has constant coefficients and it can easily be solved analytically [6]. Using $\widehat{b}=B$ in equation (4), we find the first-order Bernoulli equation (3) that must be satisfied by $b(x)$ and $c(x)$. This equation also determines the value of the constant $B$ self-consistently. We solve the Bernoulli equation (3) in Appendix 1, where we obtain generalized forms of the second-order Cauchy-Euler equation [2, 4].

On the other hand, when $\widehat{b}$ is not a constant, the predictor does not detect closed-form solutions and then the predictor of Case 2 below must be applied. 


\subsection{Case 2: the canonical form of equation (2)}

If $\widehat{b}(x)$ is not a constant, we may still try to recast equation (2) to its canonical form [4]

$$
\ddot{u}+\widehat{q}(t) u=0,
$$

where $y(t)=u(t) \exp \left(-\frac{1}{2} \int \widehat{b}(x(t)) d t\right)$ and the coefficient

$$
\widehat{q}(t)=1-\frac{\widehat{b}^{2}(t)}{4}-\frac{1}{2} \frac{d \widehat{b}(t)}{d t}
$$

recast in terms of $x$, takes the convenient form

$$
\widehat{q}(x)=1-\frac{\widehat{b}^{2}(x)}{4}-\frac{\widehat{b}^{\prime}}{2 \sqrt{c(x)}}
$$

where $\widehat{b}^{\prime}=d \widehat{b} / d x$. In this case, equation (5) can be solved easily if $\widehat{q}$ is a constant, say, $\widehat{q}=1-Q$. Then equation (7) takes the form

$$
\widehat{b}^{\prime}=2 \sqrt{c}\left(Q-\frac{1}{4} \widehat{b}^{2}\right)
$$

In the cases where the first predictor (equation (3)) fails, then equations (4) and (8) describe the second predictor, a system of two first-order equations. Eliminating $\widehat{b}$ between these two equations, we find a complicated second-order differential equation that must be satisfied by $b(x)$ and $c(x)$, viz.

$$
c c^{\prime \prime}-\frac{5}{4}\left(c^{\prime}\right)^{2}+\left(2 b^{\prime}+b^{2}\right) c^{2}=4 Q c^{3},
$$

where $^{\prime}=d / d x$. Because of the complexity in this equation, it seems to be more convenient using the two first-order equations for verification tests. We have, however, found two families of solutions of equation (9) using a new technique that we describe in Appendix 2.

The second predictor takes an analytically tractable form in the cases where the original equation (1) is already in canonical form $(b=0)$ and for $b(x)=2 /(x+C)$, where $C$ is an arbitrary constant. Then equation (9) for $c(x)$ reduces to the compact form

$$
\frac{1}{c^{3 / 4}}\left(\frac{c^{\prime}}{c^{5 / 4}}\right)^{\prime}=4 Q
$$

When this predictor succeeds, it also determines the value of the constant $Q$ selfconsistently. Equation (10) is especially useful in verification tests of many equations of interest that are in canonical form. Some notable examples are described in Section 3 below.

As was described above, for a given OSLH differential equation in canonical form, it is sufficient to test whether its coefficient $c(x)$ satisfies equation (10). Nevertheless, we may ask whether this equation can be solved analytically, in which case we can obtain the general form of $c(x)$ for which closed-form solutions are guaranteed to exist. The answer, perhaps surprisingly, is yes. We defer the general study of equation (10) to Appendix 3. 


\section{Applications of the method}

\subsection{Cauchy-Euler equation}

The Cauchy-Euler equation $[2,4]$ has $b(x)=B_{0} / x$ and $c(x)=C_{0} / x^{2}$, where $B_{0}$ and $C_{0}$ are constants. Substituting these functions into equation (4), we find that

$$
\widehat{b}=\left(B_{0}-1\right) C_{0}^{-1 / 2}=\text { const. }
$$

therefore the Cauchy-Euler equation admits analytic, closed-form solutions, as is well known $[2,4]$.

\subsection{Chebyshev equation}

The Chebyshev equation [7] has $b(x)=-x /\left(1-x^{2}\right)$ and $c(x)=N^{2} /\left(1-x^{2}\right)$, where $|x|<1$ and $N^{2}$ is a constant. Substituting these functions into equation (4), we find that

$$
\widehat{b}=0,
$$

therefore the Chebyshev equation admits analytic, closed-form solutions, as is well known $[4,7]$.

\subsection{Bessel equations of order $N$}

The Bessel equation of order $N[8]$ has $b(x)=1 / x$ and $c(x)=1-N^{2} / x^{2}$. Substituting these functions into equation (4), we find that

$$
\widehat{b}=\frac{x^{2}}{\left(x^{2}-N^{2}\right)^{3 / 2}}
$$

not a constant. Then we transform the Bessel equation to its canonical form (equation (23) in [4]), where we find that

$$
\widehat{q}(x)=1+\frac{1-4 N^{2}}{4 x^{2}} .
$$

This coefficient shows that, in general, the Bessel equation does not admit closed-form solutions since it does not satisfy equation (10). But in the special cases with orders $N=$ $\pm 1 / 2, \widehat{q}(x)$ becomes 1 and closed-form solutions do exist, as is well known $[3,8]$.

The same outcome is also obtained for the modified Bessel equation of order $N$ [8] (equation (28) in [4]) for which the coefficient of its canonical form is

$$
\widehat{q}(x)=-1+\frac{1-4 N^{2}}{4 x^{2}}
$$

that is $1-4 N^{2}=0$ for $N= \pm 1 / 2$ leading to a constant $\widehat{q}=-1$ value.

\subsection{Equations transformed to the Bessel type}

These equations have the form

$$
y^{\prime \prime}+x^{P} y=0 \quad(P=\text { const. } \neq 0,-2)
$$


and they were studied in [4]. In this case, $b(x)=0$ and $c(x)=x^{P}$. Substituting $c(x)$ into the predictor (10), we find that

$$
-P\left(1+\frac{1}{4} P\right) x^{-(P+2)}=4 Q
$$

which is satisfied for $Q=0$ and $P=-4$ (the case $P=0$ is trivial and the case $P=-2$ is a Cauchy-Euler equation). The analytic solutions found in this case are related to those found above for the $N=-1 / 2$ Bessel equation for the following reason: equation (16) can be transformed to a Bessel equation with order $N=1 /(P+2)$ and independent variable $\xi=2 N x^{1 /(2 N)}[4]$, therefore the $P=-4$ case corresponds to the Bessel case with $N=-1 / 2$ and $\xi=-1 / x$.

\subsection{CDOS equation}

The CDOS equation $[4,9]$ has $b(x)=0$ and $c(x)=C_{0} /\left(1-x^{2}\right)^{2}$ where $|x|<1$ and $C_{0}>0$ is a constant. Substituting $c(x)$ into the predictor (10), we find that

$$
Q=\frac{1}{C_{0}}
$$

therefore the CDOS equation admits analytic solutions, as was found in [4].

\section{Summary and discussion}

We have discovered two predictors (expressed by the differential equation (3) and by the combination of equations (4) and (8)) for analytic studies of all OSLH differential equations (Section 2). The latter predictor is also described by equation (9) and it is considerably simplified (see equation (10)) if the given OSLH equation is in canonical form. The first predictor is applied to the general form (1), whereas the second predictor is applied to the canonical form (5) after the first predictor has returned negative results (or to the general form (1) with $b=0$ or $b=2 /(x+C)$, where $C$ is a constant). When either predictor is satisfied, the result is positive and the given OSLH equation is guaranteed to admit a transformation to a form with constant coefficients that can easily be solved in closed form.

In Section 3, we have analyzed some differential equations of wide interest in applied mathematics [1-9] and we confirmed that they possess analytic, closed-form solutions. We believe that this new diagnostic tool will prove very useful in future studies of many more OSLH differential equations of interest to theoretical physicists and applied mathematicians.

We note, however, that this method of analysis cannot detect the existence of one analytic particular solution in the cases where the other linearly independent solution cannot be written also in closed form. An example kindly provided by the reviewers is the set of equations $y^{\prime \prime} \pm x y^{\prime} \mp y=0$, which possess the particular solution $y=x$ and another particular solution that cannot be written in closed form (it contains the error function $\operatorname{erf}\left(\sqrt{x^{2} / 2}\right)$ ). In this case, both predictors determine (correctly) that the general solutions of these equations do not have closed forms, but they do not detect the solution $y=x$.

Furthermore, the transformation (2) is not appropriate and does not work for equations whose canonical form happens to have a constant coefficient. An example is the equation 
$y^{\prime \prime}+2 x y^{\prime}+x^{2} y=0$ whose canonical form is $u^{\prime \prime}-u=0$ with $y(x)=u(x) \exp \left(-x^{2} / 2\right)$, and that can be solved easily. For this reason, the transformation of the general equation (1) to its canonical form should always be attempted before the above methodology is applied.

\section{Appendix 1: Solutions of equation (3)}

Equation (3) for $c(x)$ is a first-order Bernoulli equation with index $3 / 2$. The transformation $c=1 / z^{2}$ leads to a simple linear equation for the function $z(x)$, viz.

$$
z^{\prime}-b(x) z=-B
$$

where $B$ is constant. Its integrating factor is

$$
i(x)=\exp \left(-\int b(x) d x\right)
$$

and it leads to the general solution of equation (3) of the form

$$
c(x)=\left[\frac{i(x)}{C-B j(x)}\right]^{2},
$$

where $C$ is the integration constant and

$$
j(x) \equiv \int i(x) d x
$$

Given a form for $b(x)$, these equations determine all forms of $c(x)$ for which closed-form solutions of equation (1) are guaranteed to exist.

In the special case of $b(x)=D / x$ (where $D$ is a constant) that is of wide interest and applicability, we find the following forms of $c(x)$ :

$$
c(x)=\frac{1}{x^{2}(C-B \ln |x|)^{2}} \quad(D=1),
$$

and

$$
c(x)=\frac{(D-1)^{2}}{x^{2}\left[C(D-1) x^{D-1}+B\right]^{2}} \quad(D \neq 1) .
$$

Differential equations of the form (1) with coefficients $b(x)$ and $c(x)$ such as given here are generalizations of the well-known Cauchy-Euler equation $[2,4]$ in which $b(x) \propto 1 / x$ and $c(x) \propto 1 / x^{2}$. For these generalized equations, closed-form solutions are guaranteed to exist.

Finally, we note that we have also investigated another special case of wide interest in which $b(x)=D / x^{2}$ (where $D$ is a constant) in equation (3). In this case, we have found additional forms of $c(x)$ that guarantee closed-form solutions of equation (1), but these forms are significantly more complicated than those of the previous case, viz.

$$
c(x)=\left[\frac{\exp (D / x)}{B\left\{x \exp (D / x)-D \mathcal{E}_{i}(D / x)\right\}+C}\right]^{2} \quad(D / x>0),
$$


where $C$ is the integration constant and $\mathcal{E}_{i}$ is the exponential integral defined by [3]

$$
\mathcal{E}_{i}(\zeta) \equiv-\int_{-\zeta}^{\infty} \frac{\exp (-t)}{t} d t \quad(\zeta>0)
$$

\section{Appendix 2: Solutions of equation (9)}

We have obtained a family of solutions of equation (9) using a recently developed technique [10]. In this methodology, the terms with and without derivatives are brought to balance individually, and the two partial equations are then solved simultaneously. Applying the technique to equation (9) results in two equations, viz.

$$
c c^{\prime \prime}-\frac{5}{4}\left(c^{\prime}\right)^{2}=0,
$$

and

$$
c=\frac{1}{4 Q}\left(2 b^{\prime}+b^{2}\right) \equiv \frac{A^{2}}{4 Q},
$$

where $A^{2}$ is an arbitrary constant. Since $c$ is assumed to be constant, equation (27) is satisfied identically and equation (28) takes the form

$$
2 b^{\prime}+b^{2}=A^{2}
$$

which has the general solution

$$
b(x)=A \tanh \left[\frac{A}{2}(x+C)\right]
$$

where $C$ is the integration constant. Equations (28) and (30) represent a three-parameter family of solutions of equation (9) with constant $c$. A particular solution with $A=2, C=0$, and $Q=1 / C_{0}$ (equation (18)) was first discovered by [4] in the case of the CDOS equation [9].

Another family of solutions can be obtained by assuming that $c(x)$ is not constant in which case first we solve equation (27) for $c(x)$ (we find the same form as equation (37) below), and then we solve the replacement of equation (28), viz.

$$
2 b^{\prime}+b^{2}=4 Q c(x)
$$

for $b(x)$. Unfortunately, these solutions do not appear to be useful in practice because the form of $b(x)$ is extremely lengthy and complicated and very much unlike the forms encountered in the literature [1-3]. Only when we choose $Q=0$, in which case equations (27) and (31) decouple, can we find a useful and familiar solution for $b(x)$, that is,

$$
b(x)=\frac{2}{x+C}
$$

where $C$ is the integration constant. As was shown in [4], this form of $b(x)$ represents the complete absence of damping in the fundamental equation (1) and, using also equation 
(37) for $c(x)$, the resulting undamped OSLH differential equation

$$
y^{\prime \prime}+\frac{2}{x+C} y^{\prime}+\frac{M^{2}}{(x+L)^{4}} y=0 \quad(C, M, L=\text { const. })
$$

is a generalization of the special equation (16) with $P=-4$, which was studied in Section 3.4. All equations of the form (33) admit analytic solutions in closed form.

\section{Appendix 3: Solutions of equation (10)}

Equation (10) for $c(x)$ of the canonical form $(b=0)$ of equation (1) can be written in the expanded form

$$
c c^{\prime \prime}-\frac{5}{4}\left(c^{\prime}\right)^{2}=4 Q c^{3},
$$

which has the general solution

$$
c(x)=\frac{K^{2}}{\left[Q-K^{2}(x+L)^{2}\right]^{2}},
$$

where $K^{2} \neq 0$ and $L$ are the integration constants. The CDOS case analyzed in Section 3.5 is a particular case of this solution for $K^{2}=Q$ and $L=0$. Using these values in equation (35), we obtain

$$
c(x)=\frac{1}{Q}\left(1-x^{2}\right)^{-2},
$$

which is indeed the coefficient of the CDOS equation for $Q=1 / C_{0}$, as was also found in equation (18) above.

For $Q=0$, equation (35) takes the form

$$
c(x)=\frac{M^{2}}{(x+L)^{4}} \quad(Q=0),
$$

where $M^{2}=1 / K^{2} \neq 0$ and $L$ are arbitrary constants. The $c(x)=x^{P}$ case analyzed in Section 3.4 is a particular case of this solution for $M^{2}=1$ and $L=0$. Using these values in equation (37), we obtain $c(x)=x^{-4}$, therefore $P=-4$, as was also found above from equation (17) for $Q=0$.

Equation (34) admits also a singular solution of the form

$$
c(x)=\frac{C_{0}}{x^{2}},
$$

where $C_{0} \neq 0$ is a constant, in which case we find that

$$
Q=\frac{1}{4 C_{0}} \text {. }
$$

This case corresponds to the canonical Cauchy-Euler equation of Section 3.1 with $B_{0}=0$. We see then that both methods succeed independently in predicting closed-form solutions for the Cauchy-Euler equation. 
We conclude that all OSLH differential equations $(1)$ in canonical form $(b=0)$ with coefficients $c(x)$ of the forms described by equations (35), (37), and (38) can be solved in closed form. Since all OSLH equations can be transformed to the canonical form, the results of this appendix can be used to study all such equations of interest.

\section{Acknowledgements}

We thank the referees for their suggestions that led to an improved presentation of our results. During this research project, DMC was fully supported by the University of Massachusetts Lowell and QDK was fully supported by the Jordan University of Science and Technology.

\section{Competing interests}

The authors declare that they have no competing interests.

\section{Authors' contributions}

Both authors drafted the manuscript, and they read and approved the submitted version.

\section{Author details}

${ }^{1}$ Department of Mathematical Sciences, University of Massachusetts Lowell, Lowell, MA 01854, USA. ${ }^{2}$ Department of Mathematics and Statistics, Jordan University of Science and Technology, Irbid, 22110, Jordan.

\section{Publisher's Note}

Springer Nature remains neutral with regard to jurisdictional claims in published maps and institutional affiliations.

Received: 30 March 2017 Accepted: 10 July 2017 Published online: 26 July 2017

\section{References}

1. Whittaker, ET, Watson, GN: A Course of Modern Analysis, 3rd edn. Cambridge University Press, Cambridge (1920)

2. Hartman, P: Ordinary Differential Equations. Wiley, New York (1964)

3. Abramowitz, M, Stegun, IA (eds.): Handbook of Mathematical Functions with Formulas, Graphs, and Mathematical Tables. Dover, New York (1972)

4. Christodoulou, DM, Graham-Eagle, J, Katatbeh, QD: A program for predicting the intervals of oscillations in the solutions of ordinary second-order linear homogeneous differential equations. Adv. Differ. Equ. (2016). doi:10.1186/s13662-016-0774-x

5. Boyce, WE, DiPrima, RC: Elementary Differential Equations and Boundary Value Problems, 5th edn. Wiley, New York (1992)

6. Zwillinger, D: Handbook of Differential Equations, 3rd edn. Academic Press, Boston (1997)

7. Bell, WW: Special Functions for Scientists and Engineers. Dover, New York (2004)

8. Watson, GN: A Treatise on the Theory of Bessel Functions. Cambridge University Press, Cambridge (1922)

9. Chuaqui, M, Duren, P, Osgood, B, Stowe, D: Oscillation of solutions of linear differential equations. Bull. Aust. Math. Soc. 79, 161-169 (2009) (CDOS)

10. Christodoulou, DM, Kazanas, D: The case against dark matter and modified gravity. J. Mod. Phys. 7, 680-698 (2016)

\section{Submit your manuscript to a SpringerOpen ${ }^{\circ}$ journal and benefit from:}

- Convenient online submission

- Rigorous peer review

- Open access: articles freely available online

- High visibility within the field

- Retaining the copyright to your article 